Teil 3: Haftungsrechtliche Aspekte des Off-Label-Use 


\title{
Ärzte und ihre Verordnung von Arzneimitteln im Off-Label-Use ${ }^{1}$
}

\author{
Ute Walter
}

A. Zivilrechtliche Aspekte

I. Der Rahmen: Behandlungs-Standard und Heilversuch

II. Weitgehende „Unerheblichkeit“" arzneimittelrechtlicher Zulassung 107

III. Rechtsanspruch auf Facharztstandard

1. „Etablierter“ Off-Label-Use

108

2. „Nicht-etablierter" Off-Label-Use

109

IV. Erhöhte Aufklärungsanforderungen

B. Sozialrechtliche Aspekte

I. Referenzkriterium: Arzneimittelrechtliche Zulassung in/für Deutschland

II. Referenzkriterium: Andere Indikation ?

III. Ausnahmen-Kataloge der Rechtsprechung

IV. Rechtsanspruch des Versicherten

V. Wirtschaftliche Aufklärung

VI. Finanzierung der Arzneimittelkosten nach Sektoren

C. Verfassungs- und sozialrechtliche Aspekte

D. Strafrechtliche Aspekte

E. Zusammenfassung der Ergebnisse

Schon vor einigen Jahren habe ich in einem Aufsatz den Vertragsarzt beim OffLabel-Use zwischen Skylla und Charybdis gesehen: Entscheidet er sich dafür, gerät er in die Regressfalle - verzichtet er darauf, zieht am Horizont ein Haftungsrisiko auf ${ }^{2}$. Daran hat sich - auch wenn sich seither in der Rechtsprechung Einiges getan hat ${ }^{3}-$

1 Dieser Beitrag wurde bereits, in leicht abgeänderter Form, in der NZS 10/2011, S. 361 veröffentlicht.

2 Walter, AZR 2007, S. 113 m. w. N.

3 Seit 2007: BSG, U. v. 27.03.2007, Az. B 1 KR 17/06 (Polyglobulin 10\% bei sekundär progredienter MS), bestätigt durch BVerfG, B. v. 30.06.2008, Az. 1 BvR 1665/07; BSG, U. v. 27.03.2007, Az. B 1 KR 30/06 (Cannaboide bei chronischem Schmerzsyndrom aufgrund Querschnittslähmung); BSG, U. v. 28.03.2007, Az. B 1 KR 15/07 R (Immunglobuline bei MS-Patientin mit Kinderwunsch), bestätigt durch BVerfG, B. v. 08.07.2009, Az. 1 BvR 1531/09; BVerfG, B. v. 07.04.2008, Az. 1 BvR 550/08 (Immunglobuline bei AIDS); BVerfG, B. v. 30.06.2008, Az. 1 BvR 1665/07 (Verfassungskonformität der Off-Label-Use Rechtsprechung des BSG), NJW 2008, S. 2556; BVerfG, B. v. 04.11.2008, Az. 1 BvR 2857/08 (Immunglobuline bei MS); BSG, U. v. 30.06.2009, Az. B 1 KR 5/09 R (Methylphenidat bei Erwachsenen-ADHS); BSG, U. v. 05.05.2010, Az. B 6 KA 6/09 R und 24/09 R (Arzneikostenregress wegen Verordnung von Immunglobulinen an Krebs-Patienten). 